\title{
Left in limbo: patients' views on care across the primary/secondary interface
}

\author{
Carolyn Preston, Francine Cheater, Richard Baker, Hilary Hearnshaw
}

\begin{abstract}
Objectives-To discover the views of patients about their experiences across the interface between primary and secondary health care, including referral from general practitioners, outpatient and inpatient care, discharge, and aftercare.

Design-A qualitative study involving individual and focus group interviews of patients and interviews of carers.

Subjects-33 patients who had attended at least one outpatient appointment or had been an inpatient between two and four months previously, and eight carers of patients with chronic conditions.

Setting-Three acute hospitals and one community health service in Leicestershire.
\end{abstract}

Main outcome measures-Common themes in the views of patients and carers towards their experiences of care.

Results-Five themes emerged. The first four were: "getting in" (access to appropriate care), "fitting in" (orientation of care to the patient's requirements), "knowing what's going on" (provision of information), and "continuity" (continuity of staff and coordination and communication among professionals). The fifth theme was "limbo" (difficulty in making progress through the system), which was influenced by failures in care in relation to the other four themes.

Conclusions-The concept of progress is central to patients' views of care. It involves both progress through the healthcare system and progress towards recovery or adjustment to an altered health state. Patients' views on how well they progress through the healthcare system may be an appropriate indicator for monitoring health service performance.

(Quality in Health Care 1999;8:16-21)

Clinical Audit Centre, Department of

General Practice and

Primary Health Care,

University of Leicester,

Leicester General

Hospital, Gwendolen

Road, Leicester

LE5 4PW, UK

Carolyn Preston, research

associate

Francine Cheater, senior lecturer

Richard Baker, director

Hilary Hearnshaw,

lecturer

Correspondence to:

Ms Preston.

Accepted 11 January 1999

Keywords: patients' views; primary/secondary interface

One reason for the reforms of health systems in different countries has been the need to control costs by ensuring that patients' care is managed effectively but in the least expensive settings. In the NHS in the United Kingdom (UK), costs are controlled to some degree through the general practitioner's (GP) "gate-keeping" function, although this can create problems of disjunction between primary and secondary care services. In the UK, there has been increased interest in improving coordination across the interface between primary and secondary care, and the internal market is now being developed to emphasise integration based on partnership rather than competition.

At the same time, the health service is seeking to become more responsive to the views of patients. The Patient's Charter ${ }^{2}$ and the revised complaints procedures ${ }^{3}$ are examples of this trend, and the government now plans a national survey to enable the NHS to measure itself against the expectations of its users. ${ }^{1}$ However, methods of assessing patients' views have been criticised. Often measures are not based on the values and experiences of patients themselves ${ }^{4}$ and may exaggerate the importance of amenities such as food or facilities at the expense of more important issues such as outcome and relationship with professionals. ${ }^{56}$ The lack of an accepted theory to explain what "patient satisfaction" means to patients and its implications for health care present a particular problem because it is difficult to test the validity of a measure when it is not clear what is being measured. ${ }^{78}$ Thus, before developing a measure of patients' views, the variety of those views, and the relationships between them, should be identified.

Information about The Patient's Charter This was a document issued by the Department of Health which stated that as well as the general principle of health care being available on the basis of clinical need, regardless of ability to pay, from 1 April 1992 the following would also be guaranteed for all citizens in the UK NHS:

- To be given detailed information on local health services, including quality standards and maximum waiting times

- To be guaranteed admission for treatment by a specific date no later than two years from the day when your consultant places you on a waiting list 
referred and received inpatient treatment. ${ }^{10}$ Some patients experienced difficulties caused by delays before admission or the organisation of care while they were in hospital, but this study was confined to patients undergoing hip or knee replacements. In this paper, we describe a study to identify and describe patients' views about their experiences of the healthcare system. It was the first phase of a project to develop a new instrument to measure patients' views across the interface. The findings reported in this paper were used to generate a pool of items for inclusion in the instrument. To avoid making assumptions about patients' views, we undertook a qualitative study.

\section{The College of Health}

The College of Health, based in London, is primarily a patients association and has two main functions. Firstly, to act as an information service to the general public and it provides a freephone number to this end, and, secondly, to undertake qualitative research on patients' experiences in the UK NHS.

\section{Methods}

FOCUS GROUPS AND INTERVIEWS

Focus groups and interviews were the methods of data collection. The purpose of focus groups is to "determine the perceptions, feelings, and manner of thinking of consumers about products, services or opportunities". ${ }^{11}$ Their advantage is that they enable participants to discuss and compare experiences, allowing exploration of different points of view, often leading to the generation of additional information or unanticipated topics for discussion. When attendance at a focus group was not possible, participants were offered an interview in their own home.

\section{SELECTION OF PARTICIPANTS}

Ethical approval to obtain patient lists, and to approach patients about the study with the approval of their clinician, was obtained from the local health authority ethics committee. Lists of patients who had attended their first outpatient appointment, or had been discharged from hospital between two and four months before, were obtained from the registers of three acute hospitals and one provider of community health services in Leicestershire. Seven GPs also provided lists of patients they had referred to outpatient departments between four and six months previously. Lists included basic details including age, sex, and the specialty which the patient attended or was referred to. From these lists, we selected a smaller, random sample and undertook purposeful sampling to recruit patients who might hold a range of views about their care. Patients were selected to ensure they were from a wide range of clinical specialties and there was a mix of men and women and different age groups. We had limited information about patients' socioeconomic background but postcodes were used as a guide in selecting a range of patients (the range of Jarman scores ${ }^{12}$ of patients' practices reflected this, although Jarman scores may be an insensitive measure of individual patients' socioeconomic status). Patients less than 16 years of age, emergency admissions or those attending accident and emergency (which usually involves self referral), maternity services, and those receiving palliative care were excluded. Before inviting patients to take part, GPs were asked to exclude those who were too ill.

A sample of carers of relatives with chronic health conditions including respiratory disease, renal disease, and psychiatric conditions (who met the study criteria), unrelated to the patient participants, was also identified through three local carers' groups.

Focus groups were held in an education block of one local hospital, whereas those patients unable to attend, and all carers, were offered an interview in their own homes. Letters to recruit patients were translated into Gujerati or Hindi when appropriate, and an interpreter and single sex groups were also offered. A topic guide was developed and assessed in a pilot group. The amended version (box 1) contained open questions and prompts relating to what happened and how the patient felt in relation to progress through the health system. The topic guide was also used in the individual patient interviews, and in modified form in the carer interviews. The groups were moderated by one researcher with a second as assistant. ${ }^{11}$ All interviews were undertaken by Carolyn Preston. Groups and interviews were continued until no new ideas emerged from them. All group and individual interviews were tape recorded.

\section{ANALYSIS}

All tapes were fully transcribed. Transcripts were analysed using the constant comparative method $^{13}$ to generate themes and concepts that consistently emerged within the data. Two researchers independently developed coding schemes, and any differences between them were resolved through discussion. All transcripts were then coded to support the analysis, using Ethnograph (v3.0) to assist data handling. Five main themes were identified through sorting of coded segments and graphical representation of ideas. Throughout data analysis all four researchers conferred regularly and checked the original transcripts for inconsistencies and alternatives to ensure that the process of analysis and interpretations were consistent.

\section{Results}

Six focus groups involving 28 patients were held, and five patients and eight carers were interviewed in their own homes. Table 1 shows the characteristics of the participants. The findings are presented under the five main themes that emerged from the analysis of the qualitative data of both the patients and carers. The themes are labelled in the words frequently used by patients/carers to describe their experiences. 
What do people want when they are being treated for a health problem? How do they want to feel?

What do they want from the staff?

What is it like visiting your GP? How do you feel about these visits?

What about the consultation?

What do you want from your GP?

How did you feel when you were referred by your GP?

Why did you feel like this?

Did you feel that your doctor understood you?

Did you fully understand what was happening and why you were being referred?

Would you have liked to have been given more choice in your health care?

Was there anything that you would have liked done differently?

How did you feel about your first outpatient visit?

How did your experience compare with what you expected?

How did the way you were treated by staff make you feel?

How did the non-medical staff treat you?

How did the medical staff treat you?

Was there anything you would have liked done differently?

What was it like when you were admitted to hospital?

How did you feel?

How did the way you were treated by staff make you feel?

Did you know what to expect at the hospital?

Did you feel that they understood you and your problem?

Did you fully understand what was happening?

Were you involved in decisions about your healthcare?

Was there anything that could have been done better or differently?

What happened when you were discharged? Did it go smoothly?

How did you feel?

What made you feel like this?

How did the way staff treated you make you feel?

Did you fully understand what was happening?

Do you feel you had enough information and support on being discharged?

Was there anything that you would have liked done differently?

Do you have feelings on your overall experience?

The amount of support received

Your relative's involvement

Whether the staff treating you informed each other of what was happening

Was your care a smooth process? Why did you feel this?

Box 1 Topic guide for focus groups and interviews

Table 1 Characteristics of patients and carers taking part in focus groups or individual interviews

\begin{tabular}{|c|c|c|c|c|c|}
\hline \multicolumn{6}{|l|}{ Patients } \\
\hline Age groups & Male & Female & & & \\
\hline $20-40$ & 3 & 5 & & & \\
\hline $41-60$ & 8 & 6 & & & \\
\hline $61+$ & 6 & 5 & & & \\
\hline \multicolumn{6}{|c|}{ Specialities they had been attending } \\
\hline ENT & 5 & General surgery & 5 & Orthopaedics & 5 \\
\hline Dermatology & 3 & Cardiology & 3 & Haematology & 2 \\
\hline Psychiatry & 2 & Urology & 2 & Anaesthetics & 1 \\
\hline Rheumatology & 1 & Anticoagulant clinic & 1 & Chemical pathology & 1 \\
\hline Gastroenterology & 1 & General medicine & 1 & & \\
\hline \multicolumn{6}{|c|}{ Underprivileged score ${ }^{12}$ of patients' general practices: Mean 5.6, range -16.5 to 33.7} \\
\hline Carers & \multirow{2}{*}{\multicolumn{5}{|c|}{$\begin{array}{l}5 \\
\text { mean } 4.5 \text { (range } 0.5-10)\end{array}$}} \\
\hline Years as carer & & & & & \\
\hline
\end{tabular}

(1) GETTING IN

This theme comprised responses about gaining access to appropriate care, and included obtaining appointments, being referred, hospital admission procedures, and receiving after care. When access was successfully negotiated, patients talked not only of gaining entry to the healthcare system but also about being able to begin to make progress through it:
"At that stage you can start to be treated... if you can get that out the way quickly, off you go." (patient)

Patients and carers identified various factors that facilitated or delayed access and their subsequent progress through the system. For example, the attitudes of reception staff in practices and outpatient clinics were often identified as presenting barriers, the consequences of which made patients feel "in the way" or "a nuisance".

Good patient/doctor relationships were associated with fewer barriers to referral. Patients were generally less confident about being referred, or receiving appropriate treatment, when they were unable to see their personal doctor or when the relationship with their GP was poor. The need for their problems to be recognised as legitimate was viewed by patients as essential, determining the speed with which they gained access to care. Delays in referral and treatment were often associated with chronic health problems (for example, persistent back pain), stigmatising conditions (for example, mental illness or alcohol dependency), and problems thought to be of psychological or social origin. For some patients this led to a mismatch between their expectations and the actions of their doctor:

"It's all subjective, pain in the back, so you feel that perhaps they don't believe you if they can't see it or feel it." (patient)

Gaining access to appropriate care was often accompanied by feelings of intense relief, even in circumstances when quite serious conditions had been diagnosed. Being successful at "getting in" affirmed patients' legitimacy to investigation and treatment, and enabled them to begin to make progress through the health system:

"I was in a lot of pain and was not really noticed until the person who took me said 'I think he's having a heart attack'-'oh right we'll look at you in a minute, not half an hour'. They did the tests and said 'yes you are having a heart attack'. I was quite relieved at this stage, I thought 'Oh good, I'm glad I'm not wasting your time!'” (patient)

\section{(2) FITTING IN}

This theme was concerned with staff/patient relationships, in particular the extent to which healthcare settings and routines took account of the needs of patients and the extent to which the patient had to "fit in" with the service. If doctors, nurses, and other staff were perceived as caring and responsive to an individual patient's needs, respondents talked about being comfortable, confident, and the system "fitting in" with their requirements. Responses also reflected a desire for care to be provided in ways that preserved dignity and privacy, which in turn increased confidence:

"Although a nurse has responsibility for five or six patients, they have time for you as an individual. It's all about flexibility, they will bend their system if you want to vary from it." (patient)

When care was perceived to be impersonal and organised according to the routines of staff or the organisation, patients commonly described feeling anxious, insignificant, and 
powerless. In these situations, patients described having to fit in with a system that appeared to take no account of them as people:

"I think you feel a bit like an accessory, you've got this great big medical system and you're really not part of it, the system rolls on whether you're there or not... as a patient I thought the system was there because of you, not you there because of the system. It's this great big wheel of medicine going round and round and you're an insignificant speck.” (patient)

Patients'/carers' and staff relationships were influenced by the organisational context in which they interacted. Relationships with GPs were often built up over a period of time, and, consequently, respondents often felt they had access to information and had some degree of participation in decisions about their care. In comparison, relationships with hospital doctors tended to be viewed as more impersonal, interactions being of limited duration and presenting fewer opportunities for sharing information and participating in decisions about care. A good relationship with health professionals, where trust and understanding existed, created a sense of confidence in the patient about being able to make progress within the system:

"(my GP has) got all the time in the world...he makes you feel very comfortable and as far as any ailments are concerned he is right on the ball." (patient)

(3) KNOWING WHAT's GOING ON

Patients and carers wanted understandable and consistent information, presented in an honest and sympathetic way. Not knowing what to expect produced feelings of uncertainty and anxiety. This was most evident when patients were waiting for the results of investigations or for a diagnosis to be confirmed. When appropriate, timely information was provided, patients were reassured, gained confidence, and felt they had some degree of control over what happened to them:

"...when I go in that cubicle with all the machines they (the staff) tell me exactly why they are doing it, what the outcome is, and you get confidence and you feel different, you really do." (patient)

When information was lacking, patients and carers often described feelings of "being stuck" in the system and unable to make progress:

"When I came to the (hospital) they didn't have anyone come and see you. Nobody knew what to expect, and... that was distressing people more than anything else, they knew they were going in for major surgery, but they didn't know what to expect at all." (patient)

The quality of staff/patient relationships was a major factor influencing the accessibility of information, as described in the theme fitting in. Patients and carers tended to find it easier to obtain information from their GPs, whereas in hospital settings barriers to communication were related to perceptions of lack of staff time and inequality in status between hospital consultants and their patients:

"I find the surgeon I can't understand... is above me on his level...I just wanted to sit down and ask him my questions at my own pace and he won't... he was too sort of majestic." (patient)

Lack of information perpetuated patients' feelings of an imbalance in status and power, and reduced their sense of being involved in their own care.

When information was not readily given, or perceived to be inadequate, patients or carers often used strategies to find out more, including preparing lists of questions to ask during their consultations, arguing with doctors or other staff, and getting others, particularly those with inside knowledge of the NHS, to act for them.

\section{(4) CONTINUITY}

Continuity was achieved through receiving care from a particular professional throughout the care process, and receiving consistent, coordinated care from different staff working together. Seeing the same professional made patients feel that there was someone who was interested in them and would take time to listen. Patients recognised that continuity could facilitate the progress of treatment because the professional had sufficient background information and knowledge of their case.

When care was provided across different settings, communication and coordination were crucial. Lack of consistency across settings was a frequent source of problems, for example patients receiving conflicting information from their GP and the consultant. This could prevent patients making progress and could result in reduced confidence in care providers, increasing anxiety, and feelings of not being valued as individuals. The coordination of different services, and the degree to which care continued across interfaces, were important preconditions for the smooth progress of patients through the system. Services which patients expected to be arranged were sometimes not provided or were delayed. Sometimes GPs were not informed of outcomes of their patients' treatment or care:

"Separate clinics don't talk to each other or ring each other. I find the whole thing incredible the length of time it takes; it's just been horrendous, waiting weeks to see a consultant to be told 'I don't know why you've been referred to me'. . .It can make you feel very insignificant." (patient)

(5) LIMBO

Limbo described a state in which patients felt they were not making progress and were unable to take action to progress onwards through the system. Limbo was precipitated by poor experiences in any of the four other themes. The main features that characterised the feeling of limbo were: an indefinite period of waiting, with uncertainty about what to expect or what would happen next; a feeling of being unimportant and insignificant; and a feeling of powerlessness and loss of control over what was happening:

"You're in a lot of pain and you don't know what is wrong, you have to wait till someone comes to see you for reassurance. It can be hours...you're just put in the day room, you're just left in limbo, left 
hanging, not knowing what to expect, and you feel like you're a nuisance, you're in the way." (patient)

Limbo was most often experienced as patients moved from one stage of care to another across interfaces or between professionals, or when awaiting the results of investigations or decisions about their management. At these times, they could experience feelings of discomfort and uncertainty which were exacerbated by lack of information or failures of continuity:

"I had some further tests in early fune and I have still not heard anything. I think that is one of the worst things ...you are just left in mid air."

Most respondents did not mind waiting when they had been given some idea of how long they would have to wait and when it was not disproportionate to the urgency of their problem. However, uncertainty about how long they would have to wait, or what would be happening next, often left them feeling in limbo.

Other patients described being passed back and forth repeatedly between different settings, for example between GP and outpatient clinic, without a definitive diagnosis being made or treatment initiated. These patients often described feeling "left in limbo"-incidental to the system and unable to make any progress within it:

"It seemed a long time and I didn't feel anyone was doing anything ... I felt I was being 'fobbed off' and they didn't really understand what the pain was like." (patient)

RELATIONSHIP BETWEEN THE THEMES

The central issue which emerged from the views of patients and carers is the concept of progress. Patients perceive themselves as making progress through the healthcare system, which begins with care from the GP and goes on to include outpatient, inpatient, and aftercare. From the patient's perspective, however, progress is more than a temporal sequence of events within a system; it also includes the patient's passage from illness to recovery, or if recovery is not possible, psychological and social adjustment to an altered state of health. Failures within the system may not only delay the patient's clinical progress but may also have consequences for psychological progress, one feature of which can be the feelings associated with limbo, such as anxiety and powerlessness. In contrast, efficient progress through the system leads to feelings of confidence and reassurance. The theme patients identified as limbo reflects their psychological or emotional reaction to the extent of their progress.

Failures in any of the first four themes can cause progress through the system to be delayed, and can also have a negative impact on psychological progress, giving rise to feelings of limbo. For example, patients' progress through the system can be obstructed by failures in coordination of the system, and delayed by obstructive receptionists or failure of the GP to recognise that referral is required. Even where progress through the system is not obstructed, however, lack of information or poor relationships with healthcare staff may cause patients to feel that they are failing to make any progress. Also, lack of information or poor relationships with staff can leave patients and carers feeling powerless to challenge failures in the system. Those patients who felt able to take action to resume their progress through the system and escape from limbo often relied on their carer or a friend to act on their behalf.

\section{Discussion}

We investigated the views of a group of patients and carers of their recent experiences of the healthcare system. We identified five common themes underlying these views and developed a preliminary model to describe the relationship between them. Other studies which have examined patients' views of their health care in different settings have identified themes which are similar to those of this study. ${ }^{74-18}$ For example, themes relating to access to services (getting in), ${ }^{15-17}$ orientation of care to the patient's needs (fitting in), ${ }^{16}$ information and communication (knowing what's going on), ${ }^{15-17}$ and continuity of care ${ }^{16} 18$ have been identified in both hospital and primary care. In this study these four themes emerged consistently, suggesting that regardless of setting, these dimensions of care are of central importance to patients. Previous studies have tended to ask about experiences of care in a specific healthcare setting. In our study, patients were asked about experiences through the healthcare system as a whole, which provided an insight into how experiences at one stage of care related to experiences at later stages. In particular, this study identified that poor experiences associated with any of the four themes could lead to feelings of "limbo", a state in which patients perceived that their progress through the system was prevented or delayed. The need for patients to feel they were making progress within the system was particularly important to them, and was associated with a sense of confidence and being in control.

This study was qualitative and cannot identify the numbers of patients that had particular views. Nevertheless, the model has practical implications for health providers in primary and secondary care. The findings indicate that patients experience care across the interface as a clinical and personal path or journey in which they make progress towards a particular goal. The needs of each patient in reaching their goal may be different, and the actions of professionals or organisation of the system, or both, can assist or obstruct that progress. Rather than simply focusing on clinical progress, health professionals should check whether their patients feel they are making progress, and consider what steps may be needed to promote it. For example, some patients may require additional information, some may need the professional to listen to their concerns about their illness, and some may require organisational arrangements to ensure continuity. Health professionals also need to recognise the interdependency of their roles within the health system as a whole if patients are to avoid the fragmented care that frequently leads to limbo. 
The findings also suggest that services should be organised to avoid system failures that cause patients to feel in limbo. The identification of the most appropriate organisational structures for delivering care should be investigated in studies that include measurement of patients' views. Measures that concentrate on amenities such as food and cleanliness, however, will not provide information about issues of central concern to patients. Even asking for factual reports about accessibility or continuity may fail to identify patients' feelings about their clinical and personal progress. We are using the findings to guide the development of a new measure of patients' views, in which attention is being given to patients' perceptions of progress, and the consequences of lack of progress, for example as reflected in the theme of limbo.

We are grateful to all the patients and carers who took part and the staff who facilitated access.

The study was funded by the NHS R\&D programme (primary/secondary interface), project reference 01-26.

1 Secretary of State for Health. The new NHS: modern, dependable. London: The Stationery Office Limited, 1997.

2 NHS Management Executive. The patient's charter and primary health care. EL(92)88. London: Department of primary health

3 Review Committee on NHS Complaints Procedures. Being heard (Wilson Report). London: Department of Health, 1994.
4 Avis $\mathrm{M}$, Bond $\mathrm{M}$, Arthur A. Satisfying solutions? A review of some unresolved issues in the measurement of patient satisfaction. F Adv Nurs 1995;22:316-22.

5 Cleary PD, Edgman-Levitan S. Health care quality. Incorporating consumer perspectives. $\mathcal{F} A M A$ 1997;278: 1608-12.

6 Scott A, Smith RD. Keeping the customer satisfied: issues in the interpretation and use of patient satisfaction surveys. Int 7 Quality in Health Care 1994;6:353-9.

7 Wensing M, Grol R, Smits A. Quality judgements by patients on general practice care: a literature analysis. Soc Sci Med 1994;38:45-53.

8 Baker R. Pragmatic model of patient satisfaction in general practice: progress towards a theory. Quality in Health Care 1997;6:201-4.

9 Bruster S, Jarman B, Bosanquet N, et al. National survey of hospital patients. BMF 1994;309:1542-9.

10 Hughes A, Friere A. When waiting is not a game. London: The College of Health, 1994

11 Krueger RA. Focus groups: a practical guide for applied research. Newbury Park: Sage Publications, 1988.

12 Jarman B. Underprivileged areas: In: Gray DJP, editor. The medical annual. Bristol: Wright, 1985.

13 Strauss A, Corbin J. Basics of qualitative research. Grounded theory procedures and techniques. Newbury Park: Sage Publications, 1990

14 Hall JA, Dornan MC. What patients like about their medical care and how often they are asked: a meta-analysis of the satisfaction literature. Soc Sci Med 1998;27:935-9.

15 Ware JE, Davies-Avery A, Stewart AL. The measurement and meaning of patient satisfaction. Health and Medical Care Services Review 1978;1:1-7.

16 Delbanco TL. Enriching the doctor-patient relationship by inviting the patient's perspective. Annals of Internal Medicine 1992;116:414-18.

17 Aharony L, Strasser S. Patient satisfaction: what we know about and what we still need to explore. Medical Care Review 1993;50:49-79.

18 Barr D. The effects of organizational structure on primary care outcomes under managed care. American College of Physicians 1995;122:353-9. 